\title{
SOBRE LA AUTONOMÍA UNIVERSITARIA
}

\section{ABOUT THE UNIVERSITY AUTONOMY}

\section{Patricia Fumero Vargas*}

RESUMEN

El artículo discute los principios sustantivos de la autonomía universitaria y el cambio en el concepto desde el Manifiesto Liminar hasta la forma contemporánea de entender la autonomía, la cual está basada en dos tipos: autonomía de procedimientos y autonomía sustantiva. Asimismo, se refiere al papel de las autoridades, los docentes y los estudiantes, así como a las presiones del Estado y la sociedad para demandar rendición de cuentas y transparencia en el manejo de los recursos asignados a la educación superior.

PALABRAS CLAVE: COSTA RICA * AUTONOMÍA * UNIVERSIDADES * DOCENTES * ESTUDIANTES

\section{ABSTRACT}

This article studies the substantive principles of university autonomy and the changes in the concept from the Manifiesto Liminar to the contemporary understanding of autonomy, which is based on two types: procedural autonomy and substantive autonomy. It also discusses the role of authorities, professors and students, and the pressures of the State and society and their demand for accountability and transparency in the management of resources allocated to higher education.

KEYWORDS: COSTA RICA * AUTONOMY * UNIVERSITIES * TEACHERS * STUDENTS

Centro de Investigación en Identidad y Cultura Latinoamericanas (CIICLA) de la Universidad de Costa Rica. patricia.fumero@ucr.ac.cr 
La autonomía universitaria es un tema que preocupa en la Universidad de Costa Rica y es reforzada por los cuestionamientos de ciertos sectores a las negociaciones del FEES y por los acontecimientos acaecidos en el 2010, en la sede Rodrigo Facio, cuando ocurrió el enfrentamiento entre universitarios y agentes del Organismo de Investigación Judicial (OIJ). Aunque siempre se habla sobre la importancia de la autonomía o de la necesidad de "mayor autonomía", pocas veces se reflexiona sobre lo que supone. Al conmemorar el Día de la Autonomía Universitaria declarado por las universidades públicas costarricenses mediante acuerdos en sus respectivos consejos universitarios, tal discusión es pertinente ${ }^{1}$. Por tanto, en este artículo se revisa qué es la autonomía y cómo se refleja en el quehacer de una universidad cuya filosofía es humanística.

El mismo voto de la Sala Constitucional establece que la autonomía de las universidades públicas costarricenses:

... ha sido clasificada como especial, es completa y por esto, distinta del resto de los entes descentralizados en nuestro ordenamiento jurídico (regulados principalmente en otra parte de la Carta Política: artículos 188 y 190), y significa, para empezar con una parte de sus aspectos más importantes, que aquellas están fuera de la dirección del Poder Ejecutivo y de su jerarquía, que cuentan con todas las facultades $y$ poderes administrativos necesarios para llevar adelante el fin especial que legítimamente se les ha encomendado; que pueden autodeterminarse, en el sentido de que están posibilitadas para establecer sus planes, programas, presupuestos, organización interna y estructurar su gobierno propio (Sala Constitucional de Costa Rica, 1993).

La discusión sobre la autonomía universitaria data de tiempos de San Agustín y ha sido un principio básico para la organización de

1 Acuerdo tomado por el Consejo Universitario en la sesión extraordinaria del 14 de abril de 2010, p. 17. Universidad de Costa Rica. varias de las antiguas universidades europeas como son la de Salamanca (1243), Bolonia (s. XI), París (s. XII), Oxford (s. XII) y Cambridge (s. XIII). Sin embargo, su concepción contemporánea fue introducida a partir de la reforma al sistema educativo de 1810-1811, propuesta por Wilhelm von Humboldt en Alemania. Humboldt consideraba que los intereses del Estado $y$ de la sociedad serían mejor servidos bajo el principio de autonomía de la educación superior. Afirmó la necesidad de "tener en cuenta que su intrusión [del Estado]... siempre es un impedimento y que las cosas, en principio, funcionan infinitamente mejor sin su intromisión" (Berchem, 1985: 246)². No obstante, tales principios se replantean hoy por los cambios en el sistema universitario y la ampliación de su cobertura y sus relaciones con la sociedad, la política y la economía nacional e internacional.

El principio de autonomía universitaria se ha basado tradicionalmente en su autogobierno, su normativa propia $y$ en la libertad para el diseño de los programas de estudios. Los sucesos de abril de 2010, en la sede Rodrigo Facio, recuerdan que es necesario notar que otro aspecto considerado, cuando se habla de autonomía universitaria, es la inviolabilidad del campus universitario.

Generalmente, al discutir sobre la autonomía y las relaciones entre las universidades y el Estado, estas son analizadas a través de las leyes, normativas y regulaciones; no obstante, las investigaciones contemporáneas las analizan desde la economía política de las universidades y cómo esta impacta en la autonomía (Ordorika, 2003). La misma Declaratoria de Guadalajara sobre Autonomía Universitaria de 2011, establece que es necesario "velar colectiva, sistemática y permanentemente por la preservación y vigencia del principio de autonomía universitaria dentro y fuera de las universidades" (Universidad Estatal a Distancia, 2011).

2 Sobre los principios filosóficos relacionados con la autonomía véase Immanuel Kant, Fundamentación de la crítica a la metafísica. Capítulo tercero. Recuperado de http://www.bioetica.org/umsa/produccion/kant.pdf 
En el caso de las universidades latinoamericanas, el principio de autonomía universitaria está anclado en la Reforma Universitaria de 1918, promovida por estudiantes en Córdoba, Argentina en el Manifiesto Liminar. El movimiento estudiantil buscó eliminar la intervención del Estado en el gobierno universitario $y$ con ello, cambiaron la organización de muchas universidades y el mundo cultural latinoamericano. La reforma se fundamentó en los siguientes principios: la autonomía universitaria, el cogobierno, la extensión universitaria, el acceso por concurso y periodicidad de las cátedras, la libertad de cátedra, cátedra paralela y cátedra libre, la vinculación entre la docencia e investigación, la inserción en la sociedad y la solidaridad. En ellos se ve reflejada la estructura de la Universidad de Costa Rica.

El mismo principio de autonomía reclama la autarquía financiera y una asignación presupuestaria establecida en un mínimo que no pueda ser modificado por los gobiernos de turno. Para algunos, existe mayor autonomía cuanto más posibilidad de recursos hayan para realizar las actividades sustantivas, craso error, pues la autonomía es multifactorial motivo por el cual no se puede esencializar. Por ello, no se puede simplificar la relación entre autonomía y financiamiento, en especial cuando se escuchan voces que indican que se tendrá "mayor autonomía" con el ingreso de recursos que surge de fuentes no gubernamentales. Estas sobresimplificaciones establecen, relaciones erróneas (Chiang, 2004).

El cogobierno propone que la universidad sea gobernada en forma compartida por diversos sectores de la comunidad universitaria, en específico entre docentes y estudiantes. Consecuentemente, las universidades que reclaman la autonomía promueven las organizaciones estudiantiles. Este punto fue reiterado en la Declaratoria de Guadalajara sobre la Autonomía Universitaria, al establecer que "El movimiento estudiantil es pilar fundamental de la autonomía universitaria, reconocemos y garantizamos su independencia de funciones, de organización, financiera y de gobierno" (Unión de Universidades de América Latina, 2011)
En la Universidad de Costa Rica, las autoridades son electas por su cuerpo docente $y$ sus representantes estudiantiles, como se realizo con la elección del actual rector, forma que distancia, en principio, el proceso deliberativo de la injerencia de fuerzas políticas externas. No obstante, se espera que la Universidad asuma una responsabilidad colectiva frente al terreno político y social por las decisiones que toma.

En cuanto al principio de extensión universitaria, se pretende la devolución de los resultados de su labor a la sociedad civil, de tal forma que siempre exista una relación entre el quehacer universitario $y$ la sociedad; para lograrlo las universidades crean cursos y programas de extensión y acción social. En el caso de la Universidad de Costa Rica, la extensión docente busca brindar "servicios especiales a la comunidad, tales como asesorías especializadas, servicios técnicos y de laboratorio; los cuales son dirigidos a sectores específicos con el propósito de colaborar con su desarrollo y el del país en general" (Universidad de Costa Rica, s.f.). En este aspecto, es fundamental el vínculo entre la docencia, la investigación y la extensión docente y cultural.

La inversión de la sociedad en la educación universitaria hace que el Estado sienta la necesidad de solicitar rendimiento de cuentas y transparencia en el manejo de los recursos, para asegurar que son utilizados en forma eficiente. Otra fuente de presión para el rendimiento de cuentas, es la tendencia hacia la "academización" de las actividades profesionales, las cuales han fomentado el interés del público general sobre el "entrenamiento profesional" que se brinda en las universidades públicas. No obstante, son múltiples los ejemplos en la historia en los cuales es evidente que la autonomía de las universidades no había garantizado el óptimo cumplimiento de sus tareas, es decir, la ampliación y la transmisión del conocimiento humano (Berchem, 1985: 247).

La reforma universitaria de 1918, también procuró el acceso a los puestos docentes mediante concursos abiertos y evaluación periódica. Lo anterior supone que las cátedras son ocupadas mediante concursos de oposición y antecedentes, así como son revalidadas 
periódicamente para garantizar la calidad del docente-investigador, lo que garantiza a la sociedad un nivel adecuado del rendimiento de su cuerpo docente. A tal principio se une la libertad de cátedra para investigar y enseñar, sin supervisión académica; la cátedra paralela ofrece a los estudiantes la posibilidad de elegir libremente entre ellas y la cátedra libre es el derecho de los intelectuales idóneos de difundir su conocimiento. Los tres principios garantizan a la comunidad el acceso a diversas corrientes de pensamiento y tendencias de carácter científico. Si la autonomía universitaria basada en la libertad de cátedra y de auto-gobierno se asienta en los docentes, debemos cuestionar el papel que la academia ha jugado en este proceso, pues hoy se establece que la autonomía también está en reconocer que los actores universitarios también son agentes de toma de decisión política de la vida institucional, por lo que los docentes tienen una cuota de responsabilidad por lo que sucede al interior de ella.

A dichos principios se une la importancia de abrir el acceso al conocimiento a amplios sectores, en especial, en aquellos con menor capacidad económica. De tal forma, se ha defendido el acceso universal a la educación en las universidades públicas, con base en una educación gratuita, lo cual propicia procesos de democratización ${ }^{3}$. Tal objetivo solo se logra a través del cambio de estructuras, pues la ampliación y flexibilización de los procesos de matrícula, lo cual deviene en más docentes y mejor infraestructura, traen problemas en las áreas de calidad docente y rendición de cuentas, evidenciando una lenta respuesta de la institucionalidad a los cambios y a las presiones externas, tanto por parte de oligarquías académicas como de la burocracia institucional. Así:

La responsabilidad de las universidades sobre la que se erige su Autonomía,

3 Para ampliar sobre el tema revisar Henkel, Mary. "Academic identity and autonomy in a changing policy environment". Higher Education 49 (1-2). Universities and the Production of Knowledge, 2005: 155-176. En: <http://www.jstor.org/stable/25068062> emana del reconocimiento de la educación como un bien público social, como un derecho humano, universal $y$ un deber del Estado, tal como lo señala la unEsco.

La Universidad tiene la función compleja, dada su naturaleza, de generar conocimiento, custodiarlo y socializarlo. Significa profundizar la investigación académica y la transferencia tecnológica, cultivar las artes y las letras en su máxima expresión, analizar y criticar, con objetividad, conocimiento y racionalidad. La Autonomía significa que las universidades gozan de independencia para el desempeño de sus funciones $y$ de plena capacidad jurídica para adquirir derechos y contraer obligaciones, así como para darse su organización y gobierno propios. Preservar y cuidar la libertad de decidir, cómo se va a organizar, cómo va a funcionar, qué ofrecer a la sociedad, qué pensamientos generar, qué temas de discusión y cuáles principios y valores fomentar, cómo va a usar los recursos que tiene, es lo que caracteriza fundamentalmente a una universidad con Autonomía plena (Universidad Estatal a Distancia, s.f.).

Desde la Reforma de Córdoba de 1918, los cambios en el contexto en el cual está inserta la educación superior han variado la forma en que se toman decisiones autónomas $y$ el mismo concepto de autonomía en las universidades. Hoy no es suficiente pensar en el incremento del presupuesto, en cambios en el currículo o asuntos vinculados con el profesorado, pues la nueva forma organizativa a partir de la década de 1980, está atravesada por una visión empresarial de las universidades, por lo cual se habla de "toma de decisiones estratégicas" en forma transdisciplinaria y la corporativización de la vida académica e institucional. Pese a que el cambio en las culturas universitarias inició hace más de tres décadas, la respuesta de muchas de las estructuras existentes, tales como la forma en que opera el congreso universitario y otros 
comités académicos, no corresponde a los cambios políticos y económicos ${ }^{4}$. El problema se agrava al haber menor interés de los docentes en participar en el gobierno académico, lo cual limita la opción de contar con personas idóneas en puestos de mando.

En términos generales, desde finales de la década de 1980, las universidades enfrentan cambios consonantes con las transformaciones económicas: reforma institucional, evaluación sistemática, transparencia, rendición de cuentas y diversificación en el currículo, entre otros (Capano, 2008). No obstante, la organización interna ha variado poco, lo que ha producido un desfase en su desarrollo como se ha discutido anteriormente. Hoy, una mayor autonomía universitaria está vinculada con una política de rendición de cuentas y mayor productividad académica.

Las universidades públicas latinoamericanas deben enfrentar constantes amenazas, provenientes de los intereses de grupos de poder económico y político. Hoy, la agresión a la Autonomía no solo proviene de la represión policiaca y militar, sino del deterioro de los fondos para su financiamiento, en el que subyace una tendencia a la privatización de la investigación, la docencia y la acción social, así como de condicionamientos por parte de organismos financieros internacionales (Universidad Estatal a Distancia, s.f.). El financiamiento de la investigación no debe condicionar el rumbo de la generación del conocimiento; de lo contrario, sería lesionar la autonomía. La productividad académica supone no solo un mayor ascenso en los escalafones universitarios en el ámbito profesional, individual y colectivo, sino que un buen rendimiento deviene en mayor acceso a fondos para la investigación y la acción social, junto al reconocimiento nacional e internacional. De allí que varias agendas de investigación estén diseñadas de acuerdo con la oferta de fondos existente.

$4 \quad$ Para ampliar sobre las formas de gobierno véase: Kezar, Adrianna y Eckel, Peter D. "Meeting today's governance challenges: a synthesis of the literature and examination of a future agenda for scholarship". The Journal of Higher Education 75 (4). 2004: 371-399. En: <http://www.jstor.org/ stable/3838739>
Históricamente los docentes universitarios, hasta cierto punto han organizado autónomamente sus deberes institucionales, así como en otras universidades del mundo, los docentes de la Universidad de Costa Rica se han comportado como servidores públicos "independientes, en tanto que a partir de las negociaciones que hacen con sus superiores, determinan el horario y carga académica sin tomar en cuenta las necesidades prácticas de los estudiantes o de la institución. Lo anterior es evidente cuando se establece la oferta académica en disonancia con las necesidades $y$ vivencias del estudiantado.

Asimismo, algunos docentes trabajan en otros sitios, brindan asesorías, consultorías o incluso desarrollan otra carrera fuera de la universidad, en muchos casos utilizando los recursos institucionales; sin embargo, no comparten las ganancias pecuniarias y simbólicas con la Universidad (Capano, 2008: 500). Ejemplo de ello, es la necesidad de asegurar los derechos de propiedad intelectual y la forma en que se privilegian aquellas investigaciones que los producen. Tales prácticas promueven un involucramiento del personal en el ámbito político nacional e internacional, lo cual pone en jaque la autonomía universitaria. No se tienen datos del número de profesores que están en puestos políticos en este momento, lo cual también supone un grado de conflicto de intereses en cuanto afectan directamente las decisiones que se toman al interior de la Universidad.

La reforma al sistema promovido por algunos sectores, busca evitar dichos conflictos, en especial aquellos producto de la creación de agendas para apoyar la labor privada o celo disciplinario, motivo por el cual muchas de las oligarquías académicas se resisten (Capano, 2008: 483). Un ejemplo es el reciente caso de la Facultad de Educación de la Universidad de Costa Rica y su respuesta a la reforma integral para la formación de docentes que surgió de una discusión colegiada y fue propuesta por la Vicerrectoría de Docencia 5 .

Según la investigadora taiwanesa LiChuan Chiang, otra alternativa para entender

$5 \quad$ La Resolución VD-R-8782-2012 se encuentra disponible en: http://vd.ucr.ac.cr/joomla/index.php/ resoluc/doc_download/299-vd-r-8782-2012 
la forma en que una universidad ejerce su autonomía, es identificando los poderes de toma de decisiones sobre los propios asuntos que ellas tienen (2004: 191). La autora los resume en cuatro dimensiones básicas: académica (capacidad de brindar acceso a la educación y libertad de cátedra), recursos humanos (escogencia de sus docentes y cuerpo administrador $y$ administrativo), financiera y gobierno institucional. De esta manera, en la actualidad se habla de dos tipos de autonomía: primero, la "autonomía de procedimiento" que refiere al poder de las universidades para determinar el cómo de la academia, es decir, las técnicas utilizadas para lograr los objetivos y actividades propias de la institución y segundo, de la "autonomía sustantiva", que es el poder para determinar el qué de la academia basada en los objetivos, políticas y programas institucionales. Las investigaciones enfatizan en la importancia de la autonomía sustantiva, debido a que los cambios políticos y financieros limitan el quehacer universitario al demandar transparencia en el ejercicio de sus funciones sustantivas y la rendición de cuentas ${ }^{6}$.

Entre los cambios que inciden en el quehacer de las universidades costarricenses y que corresponden a la tendencia mundial, destaca la creación del Consejo Nacional de Rectores (CONARE, 1974, reformado en 1982) y la reciente fórmula de negociación del presupuesto, el Fondo de Estímulo a la Educación Superior (FEES, 1976, primer convenio firmado en 1988); sin embargo, las estructuras internas han cambiado poco, lo que hace difícil el manejo de fondos, en especial provenientes de fondos concursables externos. Estos cambios han fortalecido la autonomía de las universidades públicas frente al Estado, al tiempo que les ha obligado a rendir cuentas en cuanto a las decisiones relativas a los programas de las carreras,

$6 \quad$ Para ampliar véase: Berdahl, Robert O. "Shared academic governance and external constraints". Organization and academic governance in higher education. Peterson, Marvin W.; Chaffed, Ellen E. y White, T.H. (eds.). Needham Heights, MA: Ginned Press, 1991. Ordorika, Imanol. "The limits of University Autonomy: power and politics at the Universidad Nacional Autónoma de México". Higher Education 46 (3). 2003: 361-388. En: <http://www.jstor.org/stable/3447508> el profesorado (mayores requisitos académicos establecidos en los concursos para el ingreso en régimen) y en el uso de recursos financieros. Tales cambios han promovido un viraje hacia la adopción de estructuras, mecanismos y valores empresariales y corporativos. Precisamente, los intereses económicos y políticos hicieron que las universidades públicas costarricenses enfatizaran en tal proceso.

Así, las instancias de toma de decisiones trans-institucionales como es el CONARE e instancias como las vicerrectorías (creadas en 1974), hace décadas empezaron a racionalizar e indirectamente dirigir las agendas de investigación y operación institucional hacia tales estructuras. De allí que la cultura universitaria haya cambiado de una agenda centrada en lo estrictamente académico, hacia una agenda que incluye prácticas administrativas basada en la racionalización de los recursos. Lo anterior supone un trabajo colaborativo, transdisciplinario e innovador basado en redes que traspasan los departamentos disciplinarios, instituciones, sectores y el Estado.

Esto se evidencia en la creación de estructuras organizacionales complejas, la creación de consejos académicos o de apoyo y unidades de vínculo externo que promueven nuevas políticas y nuevas fórmulas organizativas para determinar los currículos de forma interdisciplinaria. En suma, los procesos contemporáneos han desarrollado problemáticas nuevas:

Mientras que el desarrollo académico es el objetivo declarado de los líderes institucionales, instituciones de educación superior se han convertido en la sede de múltiples organizaciones profesionales. La academia se ha convertido en un lugar de lucha entre los académicos y otros grupos de interés, para el control de cuestiones que anteriormente se consideraban prerrogativa académica. El trabajo académico y sus relaciones se han burocratizado y visualizado. Su actuación está abierta al escrutinio administrativo interno, así como académicos $y$ la seguridad de la tenencia de un puesto docente depende de su rendimiento en 
una forma cada vez más creciente. La institución tiene más poder para afectar la vida y el trabajo académico... (Henkel, 2005: 164) $)^{7}$.

Lo anterior deviene en diversos enfrentamientos entre aquellos anclados a las antiguas formas de concebir la universidad y su quehacer y los que han desarrollado su identidad académica en los nuevos contextos, en especial porque la universidad ha dejado de ser exclusivamente una comunidad de académicos para convertirse en un servicio público con tintes empresariales y corporativos. Así, el clima para el desarrollo profesional, en los ámbitos docentes y de investigación es cada día más complejo $y$ heterogéneo. Ya no es posible pensar que cada docente-investigador controla su propio destino, pues está integrado en un contexto en el cual cada día más investigaciones son llevadas a cabo dentro de dominios definidos por múltiples intereses, tanto en el ámbito nacional como internacional. La participación de estos en el desarrollo y la revisión es a menudo permanente, en la cual, la autoridad científica puede ser una fuente decisiva de influencia. En suma, la autonomía también está relacionada con la identidad académica ${ }^{8}$.

Pensar en la autonomía universitaria obliga a contextualizarla tanto social, económica, demográfica como políticamente, debido a que la naturaleza de las relaciones entre el gobierno $y$ las universidades son cambiantes. Ahora:

La autonomía es vista como una necesidad para que las universidades cumplan adecuadamente su misión. A menudo se define como el poder de la universidad para gobernar sus propios asuntos sin interferencia externa. Tal definición, sin embargo, ignora la complicada naturaleza de la autonomía. Mientras tanto, el

$7 \quad$ Traducción propia.

8 Para ampliar sobre la formación de la identidad académica véase: Henkel, Mary. "Academic identity and autonomy in a changing policy environment". Higher Education 49 (1-2). Universities and the Production of Knowledge, 2005: 172. En: <http:// www.jstor.org/stable/25068062> grado de autonomía universitaria no sólo depende de cuánto espacio de autogobierno se deja a la universidad, sino también de la capacidad que una universidad tiene para cumplir con su misión. Por lo tanto, la imposición de un conjunto de criterios para medir la autonomía universitaria en diversos países y afirmando que los resultados son la realidad de la autonomía universitaria puede ser un procedimiento dudoso (Chiang, 2004: 191) ${ }^{9}$.

Por tales motivos, existen fuertes presiones sobre las comunidades académicas para que cambien sus estructuras con el objeto de administrar el entorno político y para revisar sus suposiciones acerca de los roles, las relaciones y los límites impuestos por los nuevos contextos a la autonomía (Henkel, 2005: 159).

Las siguientes palabras finales se toman de la Declaratoria de la Conferencia Regional sobre Políticas y Estrategias para la Transformación de la Educación Superior en América Latina y el Caribe, organizada por el Centro Regional de la UNESCo para la Educación Superior en América Latina y el Caribe, realizado en Caracas, Venezuela, en 1997, la cual indica:

El conocimiento sólo puede ser generado, transmitido, criticado y recreado, en beneficio de la sociedad, en instituciones plurales y libres, que gocen de plena autonomía y libertad académica, pero que posean una profunda conciencia de su responsabilidad $y$ una indeclinable voluntad de servicio en la búsqueda de soluciones a las demandas, necesidades y carencias de la sociedad, a la que deben rendir cuentas como condición necesaria para el pleno ejercicio de la autonomía. La educación superior podría cumplir tan importante misión en la medida en que exija a sí misma la máxima calidad, para lo cual la evaluación continua y permanente es un valioso instrumento (Universidad Estatal a Distancia, s.f.).

$9 \quad$ La traducción es de la autora. 
En suma, hoy la autonomía universitaria está anclada a la capacidad que la institución tiene para cumplir sus acciones sustantivas con transparencia frente a la sociedad.

\section{BIBLIOGRAFÍA}

Berchem, Theodor. "University Autonomy: illusion or reality?". Oxford Review of Education 11 (3). 1985. En: <http://www. jstor.org/stable/1050175>

Capano, Gilibert. "Looking for serendipity: the problematical reform of government within Italy's Universities". Higher Education 55 (4). 2008: 481-504. En: <http://www.jstor.org/stable/29735197>

Chiang, Li-Chuan. "The relationship between University Autonomy and funding in England and Taiwan". Higher Education 48 (2). 2004: 189-212. En: <http://www. jstor.org /stable/4151575>

Henkel, Mary. "Academic identity and autonomy in a changing policy environment". Higher Education 49 (1-2). Universities and the Production of Knowledge, 2005: 155. En: <http://www. jstor.org/stable/25068062>

Ordorika, Imanol. "The limits of University Autonomy: power and politics at the Universidad Nacional Autónoma de México". Higher Education 46 (3). 2003: 361-388. En: <http://www.jstor.org/ stable/3447508>

Reforma Universitaria de 1918. La juventud argentina de Córdoba a los hombres libres de sudamérica. Manifiesto de Córdoba. En: <http://www.
fmmeducacion.com.ar/Historia/ Documentoshist/1918universidad.htm>

Sala Constitucional de Costa Rica. Voto nro. 1313-93. 26 de marzo de 1993.

Unión de Universidades de América Latina (UDUAL). "Declaratoria de Guadalajara sobre Autonomía Universitaria". Foro Latinoamericano sobre Autonomía Universitaria: La Autonomía Universitaria hoy, Experiencias y Desafíos en América Latina y el Caribe. México: Universidad de Guadalajara (UdeG), 19 de mayo de 2011.

Universidad de Costa Rica. "Acuerdo 9". Sesión Extraordinaria del Consejo Universitario nro. 5435. Universidad de Costa Rica, abril de 2010.

Universidad de Costa Rica. "Extensión docente". En: <http://accionsocial.ucr.ac.cr/web/ed/ extension-docentel>

Universidad Estatal a Distancia. "Art. V, inciso 2". Sesión del Consejo Universitario 2087-2011. 31 de marzo de 2011. En: <http://www.uned.ac.cr/cun2/documents/ AUTONOMIAUNIVERSITARIAACUERDO.pdf>

Universidad Estatal a Distancia. Autonomía universitaria: condición de un pueblo libre. Manifiesto de las universidades públicas de Costa Rica. En: <http://www. uned.ac.cr/cun2/CUapoyamanifiestosobreAutonomiaUniversitaria.shtml>

Fecha de ingreso: 17/04/2012 Fecha de aprobación: 01/06/2012 